\title{
VARIABLES SOCIO AFECTIVAS Y LA EFICACIA EN LA LABOR DOCENTE
}

\author{
Heriberto Rodríguez-Mateo \\ heriberto.rodriguez@ulpgc.es \\ Davinia Saray García Silva \\ José Carlos Rodríguez-Trueba
}

Fecha de Recepción: 21 Julio 2018

Fecha de Admisión: 1 Octubre 2018

\section{RESUMEN}

Es evidente que existe un alto índice de malestar en la profesión docente. Diferentes tipos de variables (individuales, sociales y organizacionales) influyen en el profesorado haciendo que éste se tenga que enfrentar a muchas situaciones con alta incertidumbre. Las consecuencias son el estrés laboral crónico, el síndrome de desgaste profesional, o burnout y el mobbing.

Maslach y Jackson (1981) establecen unos criterios de evaluación de dicho síndrome. En el año 1996 se construyó la Escala MBI-GS por Schaufeli, Leiter, Maslach y Jackson para medir burnout en todo tipo de trabajos independientemente de las tareas que en él se realicen. Fue adaptada a la versión española por Salanova, Schaufeli, Llorens, Grau y Peiró, en el año 2000. El MBI-GS se compone por tres dimensiones: agotamiento, cinismo y eficacia profesional.

Por otro lado, en los últimos años son muchos los estudios que están utilizando el marco teórico de los Moldes Mentales, como factor explicativo de la conducta humana en distintos ámbitos. Esta teoría permite evaluar de forma operativa las distintas formas de entender el mundo y la vida, y con alta validez predictiva, siendo capaz de predecir la adaptación o el bienestar subjetivo, el éxito 0 el fracaso de la escolaridad general, las matemáticas o el ajedrez en mayor proporción que el $\mathrm{Cl} u$ otro indicador (Hernández, 2005; Hernández-Guanir y Rodríguez-Mateo, 2006).

Este estudio tiene como finalidad explorar aquellas variables socio afectivas que pueden estar relacionadas con los factores que definen el síndrome de desgaste profesional. Se parte con la idea de que para ser un docente eficaz es necesario que el individuo cuente con una serie de moldes mentales que lo sustenten en su labor profesional diaria.

Para ello, se ha llevado a cabo este estudio en un centro educativo de secundaria, con una muestra de 40 docentes a los que se les ha valorado con la ayuda de dos instrumentos: el MBI-GS y el Test de Estrategias Cognitivo-emocionales MOLDES (Hernández-Guanir, 2010). Los resultados apoyan las hipótesis iniciales que marcan una correlación positiva entre las variables socio afectivas del profesorado y su eficacia como docentes. También se han realizado diversas regresiones lineales múltiples cuyos resultados muestran que es posible obtener modelos predictivos exploratorios 


\section{VARIABLES SOCIO AFECTIVAS Y LA EFICACIA EN LA LABOR DOCENTE}

de dos de las dimensiones del MBI-GS (cinismo y eficacia) a través de los moldes más significativos que arroja el modelo, lo que confirma la validez predictiva de la teoría de los Moldes Mentales.

Palabras clave: variables socio afectivas; eficacia docente; supervisión; moldes mentales; burnout

\section{ABSTRACT \\ AFFECTIVE PARTNER VARIABLES AND EFFECTIVENESS IN TEACHING WORK}

It is evident that there is a high rate of discomfort in the teaching profession. Different types of variables (individual, social and organizational) influence teachers, causing them to face many situations with high uncertainty. The consequences are chronic work stress, burnout syndrome, and burnout and mobbing.

Maslach and Jackson (1981) establish criteria for the evaluation of this syndrome. In 1996 the MBI-GS Scale was built by Schaufeli, Leiter, Maslach and Jackson to measure burnout in all types of work regardless of the tasks performed in it. It was adapted to the Spanish version by Salanova, Schaufeli, Llorens, Grau and Peiró, in the year 2000. The MBI-GS consists of three dimensions: exhaustion, cynicism and professional efficiency.

On the other hand, in the last years many studies are using the theoretical framework of the Mental Molds, as an explanatory factor of the human behavior in different areas. This theory allows to evaluate in an operative way the different ways of understanding the world and the life, and with high predictive validity, being able to predict the adaptation or the subjective well-being, the success or the failure of the general schooling, the mathematics or the chess In greater proportion than $\mathrm{Cl}$ or other indicator (Hernández, 2005, Hernández-Guanir and Rodríguez-Mateo, 2006).

This study aims to explore those socio-affective variables that may be related to the factors that define the wearer's syndrome. It starts with the idea that in order to be an effective teacher it is necessary that the individual has a series of mental molds that support him in his daily professional work.

To this end, this study was carried out in a secondary school with a sample of 40 teachers who have been evaluated with the help of two instruments: the MBI-GS and the Test of CognitiveEmotional Strategies MOLDS (Hernández-Guanir, 2010). The results support the initial hypotheses that mark a positive correlation between the socio-affective variables of teachers and their effectiveness as teachers. Several linear regressions have also been performed, the results of which show that it is possible to obtain exploratory predictive models of two dimensions of the MBI-GS (cynicism and efficacy) through the most significant models that the model gives, confirming the predictive validity Of the theory of Mental Molds.

Keywords: affective partner variables; teaching effectiveness; supervision; mental molding; burnout

\section{INTRODUCCIÓN}

El presente estudio se centra en la investigación sobre aquellas cualidades del docente, operativizadas como variables socio afectivas, que hacen del mismo un profesional más eficaz a la hora de gestionar las tareas que tiene encomendadas.

Que existe un alto índice de malestar en la profesión docente es un hecho que cada vez se vuelve más evidente. Diferentes tipos de variables (individuales, sociales y organizacionales) influyen en el profesorado haciendo que éste se tenga que enfrentar a muchas más situaciones para las que, con frecuencia, se encuentra preparado. Las consecuencias son el estrés laboral crónico, el síndrome de desgaste profesional, o burnouty el mobbing. Son las psicopatologías más registradas, hasta el punto de que el Tribunal Superior de Justicia de Cataluña (TSJC), en el año 2005, ha reconocido 
que el "síndrome del quemado" o "burn out" es un accidente laboral, en una sentencia en la que confirma la incapacidad permanente absoluta concedida a una maestra de Barcelona.

Según los estudios de Eastman (1996), los principales síntomas del burnout son insatisfacción, negativismo, aburrimiento, falta de preparación, irascibilidad, enfermedades frecuentes, problemas de memoria, depresión y cansancio. Debido a la cantidad e intensidad de esta colección de síntomas, podemos concluir que el burnout interfiere en la vida física, intelectual, social, psico-emocional y espiritual del docente. De ahí la importancia de contemplar éste y otros aspectos pertenecientes al ámbito de su desarrollo personal, como variables que interfieren con su desempeño profesional.

Fue a partir de la publicación en el año 1981 del Maslach Burnout Inventory por Maslach y Jackson cuando se establecen unos criterios de evaluación de dicho síndrome y se extiende el uso de un instrumento de medida estandarizado para la evaluación del mismo. En el año 1996 se construyó la Escala de Maslach Burnout Inventory-General Survey (MBI-GS) por Schaufeli, Leiter, Maslach y Jackson como un instrumento de medida genérico que es útil para medir burnout en todo tipo de trabajos independientemente de las tareas que en él se realicen. Fue adaptada a la versión española por Salanova, Schaufeli, Llorens, Grau y Peiró, en el año2000. El MBI-GS se compone por tres dimensiones: agotamiento, cinismo y eficacia profesional.

Por otro lado, en los últimos años son muchos los estudios que están utilizando el marco teórico de los Moldes Mentales, como factor explicativo de la conducta humana en distintos ámbitos. Esta teoría permite evaluar de forma operativa las distintas formas de entender el mundo y la vida, y con alta validez predictiva. No sólo han sido capaces de predecir la adaptación 0 el bienestar subjetivo, sino que también han podido explicar el éxito o el fracaso de la escolaridad general, las matemáticas o el ajedrez en mayor proporción que el Cl u otro indicador (Hernández, 2005; HernándezGuanir y Rodríguez-Mateo, 2006). Las últimas investigaciones sobre los moldes mentales demuestran el valor predictivo de dichos moldes en relación a la percepción subjetiva de salud en los mayores (Rodríguez-Mateo, Luján y Torrecillas, 2015), en la eficacia mediadora en mediadores escolares (Diaz-Negrín, Rodríguez-Mateo, Rodríguez y Luján, 2015), en el mantenimiento del peso corporal normalizado (Cahue, 2015) y en los factores de personalidad (Torrecillas, Rodríguez-Mateo, DíazNegrín y Luján, 2016), entre otros.

Los moldes mentales han sido definidos como los formatos de pensamiento egoimplicativo, es decir, los modos habituales con los que una persona se enfrenta cognoscitiva y afectivamente a la realidad, y con los que interpreta y valora su relación con ella. Estos moldes son construidos por los propios sujetos como consecuencia de sus predisposiciones y experiencias (Hernández, 2002). El test Moldes nos permiten identificar treinta moldes, diez dimensiones y tres encuadres. Un perfil positivo presentaría puntuaciones altas en los tres Encuadres, lo que supondría plenitud y armonía en la realización personal. Por el contrario, un perfil negativo obtendría puntuaciones bajas en cada uno de ellos, lo que sería señal de desajuste, inestabilidad emocional e inoperancia (Encuadre de Ajuste); señal de autolimitación y estancamiento (Encuadre de Optimización); así como señal de poca capacidad de disfrute, de indecisión, de escudarse en el pensamiento como defensa de la vida, abusando del análisis y de la reflexión antes o después de actuar, así como de la preocupación y de la obsesión (Encuadre de Espontaneidad Vital). Las dimensiones Focales reflejan claramente las diez grandes estrategias cognitivas. Éstas, son representativas de los tres Encuadres Focales.

\section{OBJETIVOS DE LA INVESTIGACIÓN}

Este estudio tiene como finalidad explorar aquellas variables socio afectivas que afectan a la labor docente, tanto en relación a su desempeño profesional, como en relación a su bienestar y salud personal. 
Se parte con la idea de que para ser un docente eficaz es necesario que el individuo cuente con una serie de moldes mentales que lo sustenten en su labor profesional diaria, moldes que debe haber adquirido correctamente a lo largo de su vida, y que para estar bien en el trabajo, hay que estar bien en la vida. Es decir, que las variables socio afectivas deben afectan significativamente en el rendimiento de la labor docente.

\section{MÉTODO}

El método empleado en esta investigación ha sido el hipotético - deductivo, para contrastar si existe relación entre las variables socioafectivas (Moldes Mentales) y las tres dimensiones que componen el síndrome de burnout: agotamiento, cinismo y eficacia profesional.

\section{Diseño}

El diseño empleado es de carácter descriptivo y correlacional: hemos medido dos grupos de variables, las socioemocionales y las del síndrome de burnout, con el fin de describirlas y de conocer el grado de relación entre ellas y la manera de interactuar.

\section{Muestra}

Para ello, se ha llevado a cabo este estudio en un centro educativo de secundaria, con Los participantes del estudio fueron una muestra de 40 docentes, miembros del claustro que voluntariamente quisieron participar en el mismo, respondiendo a los dos instrumentos de medida utilizados. Como se puede observar en la tabla 1, la muestra total alcanzó la cifra de 40 docentes, 23 varones y 17 mujeres.

Tabla 1

Género de la muestra

\begin{tabular}{lrrrrr}
\hline & & \multicolumn{2}{c}{$\begin{array}{c}\text { Porcentaje } \\
\text { válido }\end{array}$} & $\begin{array}{c}\text { Porcentaje } \\
\text { acumulado }\end{array}$ \\
\hline Válidos & Mujer & 17 & 42,5 & 42,5 & 42,5 \\
& Varón & 23 & 57,5 & 57,5 & 100,0 \\
& Total & 40 & 100,0 & 100,0 & \\
\hline
\end{tabular}

\section{Instrumento}

Se han utilizado dos pruebas estandarizadas con una amplia trayectoria para acercarnos a las variables que queremos medir.

Por un lado, la eficacia en la labor docente la hemos operativizado a través de los índices que recoge la Escala de Maslach Burnout Inventory-General Survey (MBI-GS), un instrumento de medida genérico que es útil para medir burnout en todo tipo de trabajos independientemente de las tareas que en él se realicen y que fue publicado en el año 1996 por Schaufeli, Leiter, Maslach y Jackson, y adaptado a la versión española por Salanova, Schaufeli, Llorens, Grau y Peiró, en el año 2000.

EI MBI-GS se compone por tres dimensiones: agotamiento, cinismo y eficacia profesional. Los ítems deben ser respondidos por los trabajadores haciendo uso de una escala de frecuencia de tipo Likert que va de cero " 0 " (nunca) a " 6 " (siempre). Altas puntuaciones en las dimensiones agotamiento y altas puntuaciones en la dimensión de cinismo unidas a bajas puntuaciones en la dimensión eficacia profesional serán indicadoras de burnout. 
En relación a la operativización de las variables socioafectivas, hemos decidido utilizar el Test de Estrategias Cognitivo-emocionales MOLDES (Hernández-Guanir, 2010), un cuestionario que consta de 87 ítems que describen comportamientos y modos de pensar ante diferentes situaciones; en los que el sujeto debe indicar su grado de acuerdo entre estas descripciones y su forma de ser. Esta prueba de medición de estrategias mentales, fue desarrollada por Pedro Hernández (2002), Catedrático de Psicología Evolutiva y de la Educación de la Universidad de La Laguna, en Canarias.

El índice de consistencia interna es 0,91 alfa de Cronbach tomando en consideración el cuestionario en su conjunto, con los que se comprueba que cada uno de los elementos que componen el cuestionario tiene que ver con las estrategias cognitivo-emocionales. La fiabilidad de las diez dimensiones focales oscila entre el 0,75 y el 0,97 alfa de Cronbach.

\section{Procedimiento}

Se le presentó al equipo directivo la propuesta del estudio y su colaboración con el mismo. El equipo directivo trasladó al claustro la propuesta y la solicitud de colaboración.

Se presentó en una Comisión de Coordinación Pedagógica las bases de la investigación y la colaboración necesaria por parte de los docentes del centro. Esta información fue transmitida al resto del profesorado a través de los jefes de departamento. Al finalizar una sesión del claustro, se aprovechó que estaban la totalidad de los docentes y se les administró los cuestionarios para su cumplimentación.

Únicamente dos profesoras del centro se negaron a colaborar con la investigación y decidieron no responder a los cuestionarios, aún teniendo la opción de hacerlo de forma anónima. Esta opción del anonimato se les proporcionó a todo el colectivo docente e hicieron uso de ella varios profesores.

\section{RESULTADOS}

Los resultados se presentan exponiendo, en primer lugar, los estadísticos descriptivos y las frecuencias para, posteriormente, pasar a detallar el análisis de regresión y el análisis discriminante efectuado en los datos obtenidos.

La puntuación mínima que los participantes han obtenido en la dimensión de Agotamiento es de 0,2 , mientras que la máxima es de 4,8. La media de esta dimensión se sitúa en 1,85 con una desviación típica de 1,12, tal y como se puede observar en la tabla 2.

Tabla 2

Resultados MBI-Agotamiento Escala

\begin{tabular}{llcccc}
\hline & Frecuencia & Porcentaje & $\begin{array}{c}\text { Porcentaje } \\
\text { válido }\end{array}$ & $\begin{array}{c}\text { Porcentaje } \\
\text { acumulado }\end{array}$ \\
\hline Válidos & Alto & 6 & 15,0 & 15,0 & 15,0 \\
& Bajo & 10 & 25,0 & 25,0 & 40,0 \\
& Muy Alto & 1 & 2,5 & 2,5 & 42,5 \\
Muy Bajo & 3 & 7,5 & 7,5 & 50,0 \\
Medio & 4 & 10,0 & 10,0 & 60,0 \\
Alto & 16 & 40,0 & 40,0 & 100,0 \\
Medio & & & 100,0 & \\
Bajo & 40 & 100,0 & & \\
Total & & &
\end{tabular}


La puntuación mínima que los participantes han obtenido en la dimensión de Cinismo es de 0 , mientras que la máxima es de 3,5. La media de esta dimensión se sitúa en 1,16 con una desviación típica de 0,87 , tal y como podemos observar en la tabla 3.

Tabla 3

Resultados MBI-Cinismo Escala

\begin{tabular}{llcccc}
\hline & Frecuencia & Porcentaje & $\begin{array}{c}\text { Porcentaje } \\
\text { válido }\end{array}$ & $\begin{array}{c}\text { Porcentaje } \\
\text { acumulado }\end{array}$ \\
\hline Válidos & Alto & 4 & 10,0 & 10,0 & 10,0 \\
& Bajo & 5 & 12,5 & 12,5 & 22,5 \\
& Muy Bajo & 5 & 12,5 & 12,5 & 35,0 \\
& Medio Alto & 13 & 32,5 & 32,5 & 67,5 \\
& Medio Bajo & 13 & 32,5 & 32,5 & 100,0 \\
& Total & 40 & 100,0 & 100,0 & \\
\hline
\end{tabular}

La puntuación mínima que los participantes han obtenido en la dimensión de Eficacia es de 2,7, mientras que la máxima es de 6,0. La media de esta dimensión se sitúa en 4,6 con una desviación típica de 0,8 , tal y como se observa en la tabla 4.

Tabla 4

Resultados MBI-Eficacia Escala

\begin{tabular}{llcccc}
\hline & Frecuencia & Porcentaje & $\begin{array}{c}\text { Porcentaje } \\
\text { válido }\end{array}$ & $\begin{array}{c}\text { Porcentaje } \\
\text { acumulado }\end{array}$ \\
\hline Válidos & Alto & 12 & 30,0 & 30,0 & 30,0 \\
& Bajo & 7 & 17,5 & 17,5 & 47,5 \\
& Muy Alto & 1 & 2,5 & 2,5 & 50,0 \\
& Muy Bajo & 1 & 2,5 & 2,5 & 52,5 \\
& Medio Alto & 9 & 22,5 & 22,5 & 75,0 \\
& Medio Bajo & 10 & 25,0 & 25,0 & 100,0 \\
& Total & 40 & 100,0 & 100,0 & \\
\hline
\end{tabular}

\section{Análisis de Regresión Lineal Múltiple}

A través del análisis de regresión múltiple se pretende estudiar la relación entre las dimensiones del MBI-GS (Agotamiento, Cinismo y Eficacia), con los moldes mentales del Test de Moldes.

Agotamiento como variable dependiente: No es posible establecer una ecuación de regresión para poder predecir la puntuación del agotamiento a partir de las variables socioafectivas.

Cinismo como variable dependiente: En la tabla 5 se puede observar el resumen del modelo por 
pasos, siendo el número 5 el que presenta una correlación más alta, 0,74, explicando un 48\% de la varianza.

Los moldes que componen este modelo son los siguientes: M6. Evaluación Selectiva Negativa, M11. Anticipación Devaluativa, M9. Focalización en la Carencia, M27. Control Emocional Anticipatorio y M5. Anticipación Aversiva.

Tabla 5

Resumen del modelo

\begin{tabular}{lllll}
\hline Modelo & $\mathrm{R}$ & R cuadrado & $\begin{array}{l}\text { R cuadrado } \\
\text { ajustado }\end{array}$ & $\begin{array}{l}\text { Error estándar de la } \\
\text { estimación }\end{array}$ \\
\hline 1 &, $444^{\mathrm{a}}$ &, 197 &, 176 &, 78732 \\
2 &, $537^{\mathrm{b}}$ &, 289 &, 250 &, 75096 \\
3 &, $630^{\mathrm{c}}$ &, 396 &, 346 &, 70131 \\
4 &, $700^{\mathrm{d}}$ &, 490 &, 431 &, 65405 \\
5 &, $742^{\mathrm{e}}$ &, 550 &, 484 &, 62284 \\
\hline
\end{tabular}
a. Predictores: (Constante), MOL-M6-T
b. Predictores: (Constante), MOL-M6-T, MOL-M11-T
c. Predictores: (Constante), MOL-M6-T, MOL-M11-T, MOL-M9-T
d. Predictores: (Constante), MOL-M6-T, MOL-M11-T, MOL-M9-T, MOL-]
e. Predictores: (Constante), MOL-M6-T, MOL-M11-T, MOL-M9-T, MOL-]
MOL-M5-T

Eficacia como variable dependiente: En la tabla 6 se puede observar el resumen del modelo por pasos, siendo el número 4 el que presenta una correlación más alta, 0,74, explicando un 50\% de la varianza.

Los moldes que componen este modelo son los siguientes: M6. Evaluación Selectiva Negativa, M17. Atribución Falta Esfuerzo, M23. Atribución Internalista Éxito y M13. Reclusión.

Tabla 6

Resumen del modelo

\begin{tabular}{lrrrr}
\hline Modelo & R & R cuadrado & $\begin{array}{c}\text { R cuadrado } \\
\text { ajustado }\end{array}$ & $\begin{array}{c}\text { Error estándar } \\
\text { de la estimación }\end{array}$ \\
\hline 1 &, $565^{\text {a }}$ &, 319 &, 301 &, 6929 \\
2 &, $634^{\text {b }}$ &, 402 &, 370 &, 6580 \\
3 &, $688^{\mathrm{c}}$ &, 474 &, 430 &, 6256 \\
4 &, $744^{\mathrm{d}}$ &, 554 &, 503 &, 5842 \\
\hline
\end{tabular}
a. Predictores: (Constante), MOL-M6-T
b. Predictores: (Constante), MOL-M6-T, MOL-M17-T
c. Predictores: (Constante), MOL-M6-T, MOL-M17-T, MOL-M23-T
d. Predictores: (Constante), MOL-M6-T, MOL-M17-T, MOL-M23-T, MOL-M13-T 


\section{Análisis Discriminante}

A través del análisis discriminante se han dividido las dimensiones del MBI-GS en dos grupos, que puntúan alto 0 que puntúan bajo, procediendo a un análisis que permita determinar cuáles son aquellos moldes significativos que nos pronosticarían la pertenencia y discriminación de los sujetos a dichos grupos.

Agotamiento como variable dependiente: No es posible establecer un análisis discriminante para poder predecir la pertenencia de los sujetos a partir de las variables socioafectivas.

Cinismo como variable dependiente: el único molde que arroja como significativo es el M4. Afrontamiento Borroso. La tabla 7 recoge el estadístico Lambda de Wilks que informa de una explicación del $21 \%$ de la varianza. Mientras que en la tabla 8 se recoge el autovalor y la correlación canónica obtenida.

Tabla 7

Lambda de Wilks

\begin{tabular}{|c|c|c|c|c|}
\hline Prueba de funciones & $\begin{array}{l}\text { Lambda de } \\
\text { Wilks }\end{array}$ & Chi-cuadrado & $\mathrm{gl}$ & Sig. \\
\hline 1 & ,791 & 8,805 & 1 & ,003 \\
\hline
\end{tabular}

Tabla 8

Autovalores

\begin{tabular}{lrrrr}
\hline Función & Autovalor & \% de varianza & \% acumulado & Correlación canónica \\
\hline 1 & $265^{\mathrm{a}}$ & 100,0 & 100,0 &, 457 \\
\hline
\end{tabular}

a. Se utilizaron las primeras 1 funciones discriminantes canónicas en el análisis.

Eficacia como variable dependiente: dos moldes, Atribución Falta Esfuerzo y Atribución Internalista Éxito explican un mayor porcentaje de la varianza, concretamente un 36\%, como observamos en la tabla 8.

Tabla 8

Lambda de Wilks y autovalor

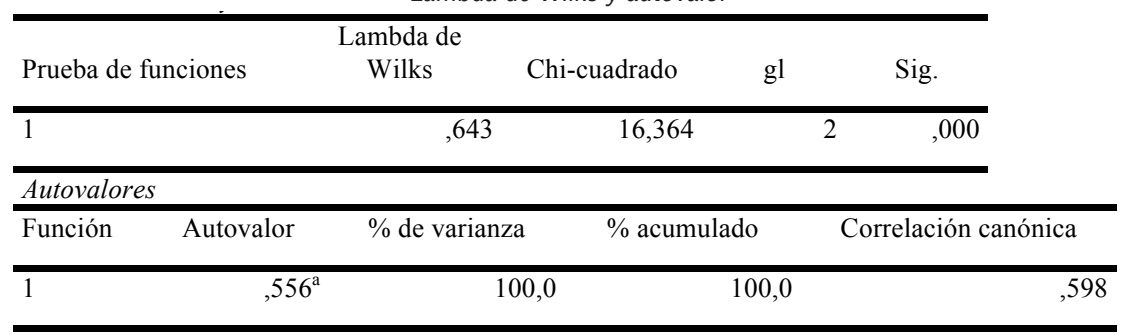

a. Se utilizaron las primeras 1 funciones discriminantes canónicas en el análisis. 


\section{DISCUSIÓN Y CONCLUSIONES}

Los resultados apoyan las hipótesis iniciales que marcan una correlación positiva entre las variables socio afectivas del profesorado y su eficacia como docentes. Las diversas regresiones lineales múltiples muestran que es posible obtener modelos predictivos exploratorios de dos de las dimensiones del MBI-GS (cinismo y eficacia) a través de los moldes más significativos que arroja el modelo, lo que confirma la validez predictiva de la teoría de los Moldes Mentales, y van en la línea de las investigaciones con moldes mentales, como las que explican la influencia de los moldes en el éxito 0 el fracaso de la escolaridad general, las matemáticas o el ajedrez en mayor proporción que el $\mathrm{Cl} u$ otro indicador (Hernández, 2005; Hernández-Guanir y Rodríguez-Mateo, 2006); el valor predictivo de dichos moldes en relación a la percepción subjetiva de salud en los mayores (Rodríguez-Mateo, Luján y Torrecillas, 2015); en la eficacia mediadora en mediadores escolares (Diaz-Negrín, Rodríguez-Mateo, Rodríguez y Luján, 2015), en el mantenimiento del peso corporal normalizado (Cahue, 2015) y en los factores de personalidad (Torrecillas, Rodríguez-Mateo, Díaz-Negrín y Luján, 2016), entre otros.

Los resultados muestran que las variables socioafectivas del test Moldes predicen dos de las tres dimensiones del MBI-GS, cinismo y eficacia, la falta de ésta, concretamente. Se han identificado los Moldes Mentales concretos que conforman el modelo predictor para cada dimensión.

Así, los moldes que forman parte del modelo predictor para la dimensión de cinismo, que explica un 48\% de la varianza, son M6. Evaluación Selectiva Negativa, M11. Anticipación Devaluativa, M9. Focalización en la Carencia, M27. Control Emocional Anticipatorio y M5. Anticipación Aversiva. Cuatro de estos cinco moldes se sitúan dentro del encuadre de ajuste, el M9, el M11, el M5 y el M6, además, estos dos últimos forman parte de la dimensión focal de negatividad. El M27 pertenece a la dimensión de optimización preparatoria dentro del encuadre de optimización.

El modelo nos arroja un perfil socioafectivo con índices que tienden a un hipersubjetivismo interferente, donde existe una alta negatividad que se manifiesta en cómo la persona juzga, anticipa e imagina la peor de las opciones. Desde ahí se construye una protección ante lo que ha de venir, por eso se le resta valor para no implicarse. Sin embargo, la insatisfacción es tal que siempre existe un deseo sobre lo que no se tiene, desconsiderando lo que sí. Este perfil comparte características con el de alguien al que catalogaríamos de cínico, lo que concuerda con el alto índice de predicción que arroja el análisis discriminante.

Por su parte, los moldes que forman parte del modelo predictor para la dimensión de eficacia, que explica un $50 \%$ de la varianza, son M6. Evaluación Selectiva Negativa, M17. Atribución Falta Esfuerzo, M23. Atribución Internalista Éxito y M13. Reclusión.

Tres de estos cuatro moldes se sitúan dentro del encuadre de ajuste, el M6, el M13 y el M17, perteneciendo cada uno a una dimensión diferente; negatividad, evitación y operatividad, respectivamente. El restante, el M23 pertenece a la dimensión de optimización autopotenciadora dentro del encuadre de optimización.

Este modelo nos arroja un perfil socioafectivo que también tiende a la negatividad, la exigencia, el enjuiciamiento, el perfeccionismo y el sufrimiento, al percibir selectivamente lo negativo de la realidad en busca de los fallos. Ante las frustraciones reacciona desde la desconexión emocional y el encierro en sí mismo, lo que provoca asilamiento, introversión y evitación. El tipo de atribuciones que lleva a cabo es internalista, ante los éxitos es debida a sí mismo y a sus cualidades, lo que le otorga autoconfianza, seguridad y autonomía; y ante los fracasos es debida a la falta de esfuerzo y dedicación, lo que se relaciona con comodidad y baja implicación y rendimiento.

En resumen, ambos modelos presentan un claro desequilibrio de las variables socioafectivas que lo componen, la mayor parte de ellas pertenecen además al encuadre que el marco teórico pre- 
senta como más importante, el de ajuste, ya que se relaciona con el bienestar subjetivo y la eficiencia.

Esta agrupación de variables socioafectivas correlaciona y permite predecir el resultado de las dimensiones de cinismo y eficacia del instrumento utilizado. Lo que nos otorga una indicación fiable del grado de burnout que pueda estar padeciendo un docente. Y, por tanto, nos marcan el camino de intervención para la creación de un programa de modificación de los moldes mentales con el objetivo de reducir el burnout a través de la mejora de la eficacia y la reducción del cinismo.

\section{REFERENCIAS BIBLIOGRÁFICAS}

Cahue. M. (2015). Estructuras cognitivas y emocionales ("Los Moldes Mentales") que intervienen en el mantenimiento del peso corporal normalizado. Tesis doctoral. Director: José Antonio Carrobles. Universidad Autónoma de Madrid, Madrid.

Cárdenas, M. (1980). Antología Supervisión Educativa. Universidad Estatal a Distancia. San José: Editorial UNED.

Chacón. F. (1990). Una empresa llamada sistema educativo. Revista del convenio Andrés Bello №14Vol.38. pp.: 99-104.

Díaz-Negrín, M., Rodríguez-Mateo, H., Rodríguez, J., y Luján, I. (2015). Valor predictivo de variables socio afectivas en mediadores escolares. International Journal of Developmental and Educational Psychology. INFAD Revista de Psicología №1-Vol.1. pp.: 265-274.

Eastman, W. (mayo, 1996). Avoiding faculty burnout through the wellness approach. Paper presented at the Annual Conference of the Association of Canadian Community Colleges. Canada.

Federación de Trabajadores de la Enseñanza de UGT. (2003). Catálogo de enfermedades profesionales de los docentes.

Hernández, P. (1991): Psicología de la Educación. Corrientes actuales y teorías aplicadas. México: Trillas.

Hernández, P. (1997). Evaluación de valores y moldes cognitivo-afectivos en la educación. En A. Cordero (Ed.), La Evaluación psicológica en el año 2000 (pp. 149-172). Madrid: TEA Ediciones.

Hernández-Guanir, P. (2002). Los Moldes de la Mente. Más allá de la Inteligencia Emocional. Tenerife, España: Tafor Publicaciones.

Hernández, P. (2005). ¿Puede la Inteligencia Emocional predecir el rendimiento? Potencial predictor de los Moldes Mentales. Revista Interuniversitaria de Formación del Profesorado №19-Vol.3. pp.: 45-62.

Hernández, P. y Rodríguez, H. (2006). Success in chess mediated by mental molds. Psicothema, №18-Vol.4. pp.: 704-710.

Hernández-Guanir, P. (2009). ¿Qué moldes mentales conforman un optimismo inteligente? Revista Interuniversitaria de Formación del Profesorado №66. Dedicado a: El optimismo. pp.: 109-127.

Hernández-Guanir, P. (2010). Manual MOLDES ( $2^{\mathrm{a}}$ ed.). Madrid: TEA Ediciones.

Hernández-Guanir, P. y Fernández-Martín, A. (2010). Mejora de los Moldes Mentales y Reducción de la Ansiedad: un Estudio Piloto. Congreso Europeo en Avances en el Tratamiento de los Trastornos por Ansiedad y Depresión. La Laguna.

Lozano, J.M. (1954). La XIX Conferencia Internacional de Instrucción Pública de Ginebra. Revista Educación №51. pp.: 16-19.

Martínez de Viergol, A. (2005). La consideración del síndrome del «Burn out» como constitutivo de la contingencia profesional del accidente de trabajo origen de la declaración de incapacidad permanente absoluta. Revista del Ministerio de Trabajo y Asuntos Sociales №59. pp.: 213-224.

Méndez, N. (1998). La supervisión y el proceso de enseñanza-aprendizaje en primaria. Revista Educación №22-Vol.2. pp.: 25-31. 
Moorer, S.H. (1969). La supervisión moderna. México D.F: Ed. Omega.

Murillo, F.J. (2005). La investigación sobre eficacia escolar. Barcelona: Octaedro.

Nando, J. (2014). Caso Práctico: Evaluación de la Práctica Docente. Revista Supervisión 21 №31. рp.: 1886-5895.

Rodríguez-Mateo, H., Luján, I. y Torrecillas, A. (2015). Aspectos socio-emocionales y salud mental y física percibidas en personas mayores. International Journal of Developmental and Educational Psychology. INFAD Revista de Psicología №1-Vol.2. pp.: 81-90.

Salanova, M., Schaufeli, W.B., Llorens, S., Peiró, J.M., y Grau, R. (2000). Desde el "burnout" al "engagement": ¿una nueva perspectiva? Revista de Psicología del Trabajo y las Organizaciones №16-Vol.2. pp.: 117-134.

Schaufeli, W. B., Leiter, M. P, Maslach, C., y Jackson, S. E. (1996). Maslach Burnout InventoryGeneral Survey. En Maslach, C., Jackson, S. E. \& Leiter, M.P (EDS.), The Maslach Burnout Inventory-Test Manual (3rd ed.). Palo Alto, CA: Consulting Psychologists Press.

Torrecillas, A., Rodríguez-Mateo, H., Díaz-Negrín, M., y Luján, I. (2016). Valor predictivo de los moldes de la mente sobre los factores de personalidad. International Journal of Developmental and Educational Psychology. INFAD Revista de Psicología №1-Vol.2. pp.: 295-306

Wiles, K. y Lovell. J.T. (1983). Supervision for better schools. New Jersey. Prentice-Hall. 
